\title{
Loudness functions with air and bone conduction stimulation in normal-hearing subjects using a categorical loudness scaling procedure
}

Stefan Stenfelt and Mehrnaz Zeitooni

\section{Linköping University Post Print}

\section{Tweet}

N.B.: When citing this work, cite the original article.

Original Publication:

Stefan Stenfelt and Mehrnaz Zeitooni, Loudness functions with air and bone conduction stimulation in normal-hearing subjects using a categorical loudness scaling procedure, 2013, Hearing Research, (301), 85-92.

http://dx.doi.org/10.1016/j.heares.2013.03.010

Copyright: Elsevier

http://www.elsevier.com/

Postprint available at: Linköping University Electronic Press

http://urn.kb.se/resolve?urn=urn:nbn:se:liu:diva-94709 


\section{Loudness functions with air and bone conduction stimulation in normal-hearing subjects using a categorical loudness scaling procedure}

Stefan Stenfelt and Mehrnaz Zeitooni

Department of Clinical and Experimental Medicine

Technical Audiology

Linköping University

58185 Linköping

Sweden

Corresponding author

Stefan Stenfelt

Department of Clinical and Experimental Medicine

Technical Audiology

Linköping University

58185 Linköping

Sweden

Tel: +46101032856

Email: stefan.stenfelt@liu.se 


\begin{abstract}
In a previous study (Stenfelt et al., 2002) a loudness balance test between bone conducted (BC) sound and air conducted (AC) sound was performed at frequencies between 0.25 and $4 \mathrm{kHz}$ and at levels corresponding to 30 to $80 \mathrm{~dB} \mathrm{HL}$. The main outcome of that study was that for maintaining equal loudness, the level increase of sound with $B C$ stimulation was less than that of $A C$ stimulation with a ratio between 0.8 and $0.93 \mathrm{~dB} / \mathrm{dB}$. However, because it was shown that $\mathrm{AC}$ and $\mathrm{BC}$ tone cancellation was independent of the stimulation level, the loudness level difference did not originate in differences in basilar membrane stimulation. Therefore, it was speculated that the result could be due to the loudness estimation procedure. To investigate this further, another loudness estimation method (adaptive categorical loudness scaling) was here employed in 20 normal-hearing subjects.
\end{abstract}

The loudness of a low-frequency and a high-frequency noise burst was estimated using the adaptive categorical loudness scaling technique when the stimulation was bilaterally by AC or BC. The sounds where rated on an 11-point scale between inaudible and too loud. The total dynamic range for these sounds was over $80 \mathrm{~dB}$ when presented by $\mathrm{AC}$ (between inaudible and too loud) and the loudness functions were similar for the low and the high-frequency stimulation. When the stimulation was by $B C$ the loudness functions were steeper and the ratios between the slopes of the $A C$ and $B C$ loudness functions were 0.88 for the low-frequency sound and 0.92 for the high-frequency sound. These results were almost equal to the previous published results using the equal loudness estimation procedure, and it was unlikely that the outcome stems from the loudness estimation procedure itself. One possible mechanism for the result was loudness integration of multi-sensory input. However, no conclusive evidence for such a mechanism could be given by the present study.

Keywords: Bone conduction, loudness, categorical loudness scaling, multi-sensory integration. 


\section{Highlights}

- The loudness function was steeper when stimulation was by BC than by AC

- The difference between the loudness function slopes was greatest at low frequencies

- The average ratios between $\mathrm{AC}$ and BC loudness function slopes were between 0.88 and 0.92

- The difference between the AC and BC loudness functions were found at both low and high intensities.

\section{Abbreviations}

AC: air conduction

ANOVA: analysis of variance

BC: bone conduction

cu: categorical unit

HL: hearing level

SD: standard deviation

SL: sensation level

SPL: sound pressure level 


\section{Introduction}

In a previous study (Stenfelt et al., 2002) the loudness of bone conducted (BC) sound was compared with that of air conducted (AC) sound. In that study, stimulation was by narrowband noises at six frequency-ranges between 0.25 and $4 \mathrm{kHz}$. The study tested a dynamic range between 30 and $80 \mathrm{~dB} \mathrm{HL}$, and the $50 \mathrm{~dB}$ increase in AC delivered sound was followed by a lesser increase of BC delivered sound for the same perception of loudness. For normalhearing participants, the greatest difference between the AC and BC level increase while maintaining equal loudness was at the lowest frequency $(0.25 \mathrm{kHz})$ where an $8 \mathrm{~dB}$ BC level increase gave the same loudness increase as a $10 \mathrm{~dB}$ AC level increase (a ratio of $0.8 \mathrm{~dB} / \mathrm{dB}$ ). At frequencies above $1 \mathrm{kHz}$, the $\mathrm{BC}$ increase to equal the loudness of an $\mathrm{AC} 10 \mathrm{~dB}$ level increase was between 9.1 and $9.3 \mathrm{~dB}$ (ratios of 0.91 to $0.93 \mathrm{~dB} / \mathrm{dB}$ ).

When the same equal loudness estimation was done with sensorineural hearing-impaired subjects in that same study (Stenfelt et al., 2002), the ratios of the BC and AC loudness functions were more pronounced at the low frequencies (below $1 \mathrm{kHz}$ ) while they were similar at and above frequencies of $1 \mathrm{kHz}$. These data indicated that there were differences between the loudness functions for AC and BC stimulation in humans.

One method that has been popular for investigating BC sound was the so-called cancellation experiments. In these experiments, a BC tone was perceptually cancelled by an AC tone of the same frequency. The first to use this method was von Békésy (1932) who was able to cancel a $0.4 \mathrm{kHz}$ BC tone by an AC tone of $0.4 \mathrm{kHz}$ in the human. That study indicated that a BC sound elicited a traveling wave on the basilar membrane that was equal to the traveling wave produced by an AC sound. In a later study, Khanna et al. (1976) explored the cancellation between AC and BC tones for levels between 40 and $70 \mathrm{~dB}$ SL. They conducted 
cancellations while altering the stimulus intensities in $10 \mathrm{~dB}$ steps; an increase in BC stimulation level of $10 \mathrm{~dB}$ resulted in a $10 \mathrm{~dB}$ level increase of the required $\mathrm{AC}$ cancellation tone. Consequently, they reported the relative amplitude and phase settings at cancellation to be independent of stimulation level.

Another AC-BC cancellation study that investigated the level-dependence of the amplitude and phase settings at cancellation was conducted by Stenfelt (2007). In that study, either a 0.7 or $1.1 \mathrm{kHz}$ BC tone was cancelled by an AC tone at the same frequency for the three levels 40, 50 and $60 \mathrm{~dB}$ HL. The results from that study indicated that the relative amplitude and phase settings between the AC and BC tone were independent of the stimulation level. Also, that study showed that the cancellation at $0.7 \mathrm{kHz}$ was not disturbed when an additional tone of $1.1 \mathrm{kHz}$ was introduced and the cancellation at $1.1 \mathrm{kHz}$ was not disturbed by introducing a $0.7 \mathrm{kHz}$ tone. Consequently, according to Stenfelt (2007), a $10 \mathrm{~dB}$ increase in BC sound level caused a basilar membrane vibration change that was similar to that caused by a $10 \mathrm{~dB}$ increase in AC sound level.

Another indication that AC stimulation and BC stimulation caused similar vibration patterns of the basilar membrane was found in human temporal bone experiments where the vibration of the basilar membrane was measured with a laser Doppler vibrometer (Stenfelt et al., 2003). In that study, the maximum vibration response for a specific position on the basilar membrane was obtained at the same frequency for both AC and BC stimulation. Even if this was not a conclusive evidence that BC stimulation excited the basilar membrane exactly as AC stimulation excited the basilar membrane, it was an indication of similar vibration patterns. Moreover, a modeling approach for understanding the basilar membrane response with BC stimulation showed similar results as the temporal bone specimen study (Kim et al., 2011). Also, electrophysiological data (Beattie, 1998) and oto-acoustic emission data 
(Watanabe et al., 2008) indicated similarity of the basilar membrane vibration pattern when stimulation was either by AC or BC.

However, there were also documented differences between AC and BC sound perception. One such difference was the ability to perceive ultrasonic sound (20 to $120 \mathrm{kHz}$ ) when presented by BC but not through AC (Hosoi et al., 1998). The mechanisms for detection of BC ultrasound was not completely clarified and several possible explanations have been provided, such as demodulation due to non-linearities in the skull bone (Haeff et al., 1963) or non-linear processing in the cochlea (Nishimura et al., 2011).

Consequently, the differences between loudness functions found in Stenfelt et al. (2002) were speculated to originate in either the loudness estimation procedure or in multi-sensory excitation during BC stimulation resulting in loudness integration. In order to further investigate the BC stimulated loudness function, the outcome of categorical loudness scaling with AC and BC stimulation is here investigated in normal-hearing subjects.

The study was approved by the local ethical committee. 


\section{Materials and Methods}

\subsection{Subjects}

Twenty normal-hearing subjects with hearing thresholds equal or better than $20 \mathrm{~dB}$ HL for both $\mathrm{AC}$ and $\mathrm{BC}$ stimulation in the frequency range 125 to $8000 \mathrm{~Hz}$ (500 to $4000 \mathrm{~Hz}$ for BC) volunteered to participate in the study. They all had a maximum interaural difference of 10 $\mathrm{dB}$ at any audiologic frequency and their mean age was 30.6 years (range 18 to 42 years); twelve were male and eight were female. They all had otologically normal ears and none had history of ear surgery.

\subsection{Test set-up}

The loudness scaling test was computerized using MatLab® ${ }^{\circledR}$ with 24-bit amplitude resolution of the sound. The output of the computer soundcard (M-Audio Transit, using 24-bit resolution and $44.1 \mathrm{kHz}$ sampling rate) was fed to a 4-channel power amplifier (Rotel RA-04 SE) where two channels were used for AC stimulation and two channels for BC stimulation. The AC stimulation was provided by a pair of Sennheiser HDA200 earphones coupled to the amplifier while the BC stimulation was provided by a pair of custom made BC transducers. The BC transducers used a balanced technology (Håkansson, 2003) to minimize distortion; each transducer was encapsulated in a plastic housing with a flat elliptic surface of 25x18 mm and a height of $10 \mathrm{~mm}$. The BC transducers were bilaterally applied to the mastoid just behind the pinnae using an elastic band providing equal static force to both transducers. The exact static force depended on the size of the skull and the range of the static force for the participants was 3 to 5 Newtons. The stimulation was binaural for both BC and AC stimulation.

Two types of stimulation were used, one low-frequency noise ( 0.6 to $0.9 \mathrm{kHz}$ ) and one highfrequency noise (3.0 to $4.0 \mathrm{kHz}$ ). These were provided by both $\mathrm{AC}$ and $\mathrm{BC}$ and, in total, 4 
conditions were tested. To avoid order effects, the test-orders were counterbalanced among the participants. The stimuli were created in MatLab ${ }^{\circledR}$ by band-pass filtering white Gaussian noise with the corner-frequencies given above using slopes of $48 \mathrm{~dB} /$ octave. The final stimulation was a 1 second noise burst with 50 ms rise and fall times. After each stimulation the participant rated the loudness using the categorical scale described in the next section.

\subsection{Categorical loudness scaling}

The categorical loudness scaling was done according to ISO:16832 (2006) in a way similar to that described by (Brand et al., 2002). The scaling procedure was conducted on a computer with 11 response alternatives (Fig 1) were each step corresponded to an increase of 5 categorical units (0 to $50 \mathrm{cu}$ ). The participant faced a computer screen with the interface shown in Fig 1, and the response was given by clicking on one of the bars (labeled or unlabeled) with the mouse button. A response of the perceived loudness was always given before the next stimulation was provided.

First, the maximum and minimum of the scale was determined (Inaudible $=0 \mathrm{cu}$; Too loud $=$ $50 \mathrm{cu})$. This was accomplished by starting the stimulation at approximately $60 \mathrm{~dB} \mathrm{HL}{ }^{1}$ and thereafter increasing the level in steps of $5 \mathrm{~dB}$ until the loudness was rated as "Too loud" (50 $\mathrm{cu})$. Then the level was decreased in $10 \mathrm{~dB}$ steps until the sound was rated as "Inaudible". All rated loudness responses and corresponding stimulation levels were stored on the computer. As a precaution and to avoid hazardous levels, the maximum allowed stimulation level was set at $100 \mathrm{~dB}$ HL for all conditions. For all the participants, this level was only reached a few times during the loudness rating and was always rated as "Too loud". Also, to avoid that the dynamic range of the test system affected the low-level stimulations, the lowest producible level was approximately $20 \mathrm{~dB}$ below the threshold for the stimuli.

\footnotetext{
${ }^{1}$ Hearing level is not defined for these stimuli but the approximate threshold for the stimuli was piloted in 5 subjects before the study and the $60 \mathrm{~dB} H \mathrm{HL}$ was $60 \mathrm{~dB}$ above these thresholds.
} 
The adaptive procedure for determining the loudness function began by assigning stimulation levels for the five labeled parts of the scale between very soft (5 cu) and very loud (45 cu) using a linear interpolation from the obtained maximum and minimum levels. The five stimulation levels were presented in random order and rated for their loudness. The levels and their rated loudness were stored and used to compute five new stimulation levels. The equation below describes the categorical scaling $F$ [in $\mathrm{cu}$ ] as a function of the stimulation level $L$ [in $\mathrm{dB}$ ] that was used to estimate the loudness function.

$F(L)=\left\{\begin{array}{c}k_{l o}\left(L-L_{\text {cut }}\right)+25, \text { for } L \text { where } c \leq 15 \mathrm{cu} \\ k_{\text {hi }}\left(L-L_{\text {cut }}\right)+25, \text { for } L \text { where } c \geq 35 \mathrm{cu} \\ \text { bez }\left(L, k_{l o}, k_{h i}, L_{\text {cut }}\right), \text { for } L \text { where } 15 \mathrm{cu}<c<35 \mathrm{cu}\end{array}\right.$

Here, $k_{l o}$ and $k_{h i}$ are the estimated slopes $[\mathrm{cu} / \mathrm{dB}]$ at the low and high stimulation levels, respectively, $L_{\text {cut }}$ is the estimated intersection level between low and high levels, and c is the categorical scaling in categorical units. To avoid a sharp knee-point at the intersection level $\left(L_{c u t}\right)$, a quadratic Bézier curve (bez) is used for the loudness function at categorical units between 15 and 35. The above equation is similar to the equation presented in Brand et al. (2002), and the $2^{\text {nd }}$ order polynomial Bézier smoothing is done according to the procedure described in Brand et al. (2002) where the details for the current Bézier smoothing can be found. The three parameters $\left(k_{l o}, k_{h i}, L_{c u t}\right)$ were fitted to the above function $F(L)$ by minimizing the mean squared error from the rated loudness in categorical scaling units and their corresponding stimulation levels $(L)$. The three estimated parameters were then used to compute a new estimate of the loudness function according to the above equation.

The categorical loudness scaling procedure continued iteratively by assigning five new stimulation levels to the five labeled loudness categories 5, 15, 25, 35, and $45 \mathrm{cu}$. In the current study, 3 iterations were used to estimate the three parameters of the loudness function according to the equation above. 


\subsection{Calibrations}

The audiometer output for the baseline hearing test was calibrated according to the standard ISO:389-1 (1998) [AC thresholds] and ISO:389-3 (1994) [BC thresholds with B71 transducer]. For the other tests, ISO:389-8 (2004) was used to estimate the stimulation level of the noises from the earphones while ISO:389-3 (1994) was used to estimate the stimulation level of the noises from the BEST BC transducer. The Sennheiser HDA200 earphones were calibrated by attaching the earphone to an ear simulator (IEC:60318-1, 2009) and measuring the output level with a Brüel and Kjær Pulse 12.0 analyzer. The BC transducers were calibrated by attaching the transducer to the artificial mastoid Brüel and Kjær type 4930 and measuring the output with the Brüel and Kjær Pulse 12.0 analyzer. It should be noted that the BEST BC transducers used for the BC testing do not conform to the data specified in the standard (e.g. a circular interface of $175 \mathrm{~mm}^{2}$ ) and the absolute values from the calibration may deviate from that obtained with a Radioear B71 BC transducer. However, the data obtained in this way gave an estimation of the stimulation levels, and only relative data were used in the analysis.

\subsection{Statistics}

Repeated measures analysis of variance (ANOVA) were conducted on the estimated parameters separately to reveal differences (if any) between AC and BC stimulation as well as between the low and high-frequency slopes. The level of statistical significance was set at $\mathrm{p}<0.05$ 


\section{Results}

\subsection{Loudness functions}

The loudness functions measured using the categorical scaling procedure are shown in Fig 2a for AC stimulation and in Fig 2b for BC stimulation. In Fig 2, the results with low-frequency stimulation are shown with a solid line and the results with high-frequency stimulation are shown with a dashed line. The loudness functions are based on the means of the three parameters $\left(k_{l o}, k_{h i}, L_{c u t}\right)$ for each condition and are shown in relation to the stimulation level (dB SPL for AC, dB re $1 \mu \mathrm{N}$ for BC). Also included in Fig 2 are the standard deviations (SD) of the loudness functions according to the parameter estimates.

The estimated loudness functions at low and high frequencies with AC stimulation (Fig 2a) were similar. The inaudible levels $(\mathrm{cu}=0)$ were on average around $10 \mathrm{~dB}$ SPL for both stimuli and the maximum levels (Too loud, $\mathrm{cu}=50$ ) were approximately $90 \mathrm{~dB}$ SPL. The SDs also were similar for the estimated loudness functions using high and low-frequency noise bursts. The loudness functions seem different when the stimulation was through BC (Fig 2b). This apparent difference originates in the different scales used for AC and BC sound: sound pressure level (dB SPL) for AC sound and force level ( $\mathrm{dB}$ re $1 \mu \mathrm{N})$ for BC sound. Consequently, different reference levels are used for the two stimulation modalities. Also, the sensitivity in force levels is different for a low-frequency BC sound than for a highfrequency BC sound while, for the frequencies used here, the sensitivity is similar in sound pressure levels for a low-frequency AC sound and a high-frequency AC sound. These issues explain the major differences between the loudness functions in Fig 2.

With the low-frequency BC stimulation, the inaudible level was around $60 \mathrm{~dB}$ re $1 \mu \mathrm{N}$ and the maximum level was close to $130 \mathrm{~dB}$ re $1 \mu \mathrm{N}$ (approximately 3.2 Newtons) while the high- 
frequency BC stimulation resulted in an inaudible level at around $30 \mathrm{~dB}$ re $1 \mu \mathrm{N}$ and a maximum level close to $105 \mathrm{~dB}$ re $1 \mu \mathrm{N}$. Also included in Fig $2 \mathrm{~b}$ was the vibrotactile threshold level for BC stimulation at $750 \mathrm{~Hz}$ on the mastoid (Brinkmann et al., 1983) [vertical dashed line].

\subsection{Dynamic range}

The data in Fig 2 were replotted in relation to the inaudible level $(\mathrm{cu}=0)$, shown in Fig 3. Here, the range between the inaudible level and the "Too loud" level was defined as the participant's dynamic range for the stimuli. This dynamic range is displayed in $\mathrm{dB}$ SL, where $0 \mathrm{~dB}$ SL equals the stimulation level at the inaudible response $(\mathrm{cu}=0)$ even though the hearing threshold was between 0 and $5 \mathrm{cu}$. When the results were rearranged in this way, the estimated loudness functions could be compared between stimulation modalities and between low and high-frequency stimulations. The data presented are averaged loudness functions based on the average of the extracted parameters $\left(k_{h i}, k_{l o}, L_{c u t}\right)$. The two loudness functions with AC stimulation fall almost on top of each other and can be difficult to distinguish in Fig 3.

It can be seen in Fig 3 that the average dynamic ranges with the two AC stimulations were similar, $81.2 \mathrm{~dB}$ for the low-frequency noise bursts and $80.3 \mathrm{~dB}$ for the high-frequency noise bursts. The average dynamic ranges for the two BC noise bursts were smaller, $71.4 \mathrm{~dB}$ and $74.1 \mathrm{~dB}$ for the low-frequency and the high-frequency stimulation, respectively. These estimated dynamic ranges were computed according to equation 1 and the differences seen in Fig 3 were caused by the differences of the estimated parameters $k_{l o}$ and $k_{h i}$. For AC stimulation, the average low-level slope of the loudness functions $\left(k_{l o}\right)$ was $0.45 \mathrm{cu} / \mathrm{dB}$ while it was $0.52 \mathrm{cu} / \mathrm{dB}$ for the low-frequency BC stimulation and $0.50 \mathrm{cu} / \mathrm{dB}$ for the highfrequency BC stimulation. The average high-level slope of the loudness functions $\left(k_{h i}\right)$ was 
$0.92 \mathrm{cu} / \mathrm{dB}$ for the low-frequency AC stimulation, $0.98 \mathrm{cu} / \mathrm{dB}$ for the high-frequency AC stimulation, $1.02 \mathrm{cu} / \mathrm{dB}$ for the low-frequency BC stimulation, and $0.99 \mathrm{cu} / \mathrm{dB}$ for the highfrequency BC stimulation.

These differences in average loudness slopes were supported by the ANOVA that revealed a statistically significant difference of the $k_{l o}$ between AC and BC stimulation $[\mathrm{F}(1,19)=11.99$, $\mathrm{p}<0.003]$ and between low and high-frequency stimulation $[\mathrm{F}(1,19)=6.44, \mathrm{p}<0.02]$ as well as for the interaction between stimulation modality (AC and $\mathrm{BC}$ ) and stimulation frequency $[\mathrm{F}(1,19)=4.47, \mathrm{p}<0.05]$. The ANOVA showed a statistically significant difference for the high-level slope $k_{h i}$ between AC and BC stimulation $[\mathrm{F}(1,19)=4.69, \mathrm{p}<0.05]$, while no statistically significant difference was found for stimulation frequency (high or low). No analysis was done of the intersection between high-level and low-level slopes $\left(L_{c u t}\right)$ as this parameter was highly dependent of the threshold levels for the different scales used in Fig 2 (SPL or $\mu \mathrm{N}$ ). Moreover, when the loudness functions were normalized in Fig 3, the normalization caused $F(0)=0$ cu and $L_{c u t}=25 / k_{l o}$. This meant that $L_{c u t}$ was not independent but a function of $k_{l o}$. Therefore, the statistical analysis was limited to the two parameters $k_{l o}$ and $k_{h i}$.

\subsection{Differences of loudness levels between AC and BC}

Another way to illustrate the differences between the loudness functions with AC and BC stimulations are shown in Fig 4. The results with low-frequency stimulation are shown in Fig 4a and the results with high-frequency stimulation are shown in Fig 4b. The data in Fig 4 are arranged as the $\mathrm{dB}$ SL stimulation level difference at equal loudness, the loudness level difference. These loudness level difference curves are plotted as a function of the AC stimulation level in dB SL. The thin lines in Fig 4 show the individual loudness level differences, and the thick lines show the average of the individual loudness level differences. Consequently, the thick solid line in Fig 4a shows the average loudness level difference 
between AC stimulation and BC stimulation for the low-frequency noise as a function of the AC stimulation level (in dB SL). The thick dash-dotted lines are the AC-BC loudness level difference plus/minus one SD. Also included in Fig 4, as thick dashed lines, are the estimates of loudness level difference between AC stimulation and BC stimulation from Stenfelt et al. (2002), in Fig 4a the $750 \mathrm{~Hz}$ data and in Fig 4b the $4 \mathrm{kHz}$ data.

It should be noted that the stimulation ranges differed between this study (approximately 10 to $90 \mathrm{~dB}$ SPL) and the Stenfelt et al. (2002) study (30 to $80 \mathrm{~dB}$ HL). Therefore, the overall average ratios between BC loudness level and AC loudness level from the Stenfelt et al. (2002) study were used for the inclusion in Fig 4. These ratios served as slopes for the Stenfelt et al. (2002) curves in Fig 4 using a loudness level difference between AC and BC stimulation of $0 \mathrm{~dB}$ at $0 \mathrm{~dB}$ SL. In Fig 4, the slope for the average AC-BC loudness level difference with low-frequency stimulation from the current study was $0.12 \mathrm{~dB} / \mathrm{dB}$ [the mean slope at $750 \mathrm{~Hz}$ from Stenfelt et al. (2002) was $0.12 \mathrm{~dB} / \mathrm{dB}$ ]. This means that a $10 \mathrm{~dB}$ increase in AC stimulation was, on average, accompanied by an $8.8 \mathrm{~dB}$ increase in BC stimulation for equal perception of loudness. With high-frequency stimulation, the slope for the average AC-BC loudness level difference in Fig $4 \mathrm{~b}$ was on average $0.08 \mathrm{~dB} / \mathrm{dB}$ [the mean at $4 \mathrm{kHz}$ from Stenfelt et al. (2002) was $0.09 \mathrm{~dB} / \mathrm{dB}$ ]. 


\section{Discussion}

The results in this study using an adaptive categorical loudness scaling procedure indicated that the loudness functions were steeper for BC stimulation than for AC stimulation, more so with a low-frequency stimulation than with a high-frequency stimulation. These results corroborated the findings in Stenfelt et al. (2002) where a different method for investigating the loudness function of AC and BC sound was used. The use of different methods in the two studies indicated that the results were not due to the method itself, as a bracketing method was used in the Stenfelt et al. (2002) study and an adaptive categorical scaling method in this study. The adaptive categorical loudness scaling used here forced the test of AC and BC stimulation to be sequential. This avoided simultaneously AC and BC stimulation that could introduce bias from the occlusion effect (Stenfelt et al., 2007) when an earphone was used during BC stimulation. Moreover, because the order of stimulation modality (AC and BC) was counter-balanced among the participants, the results were unlikely an effect of test order.

One difference in the method between the current study and the Stenfelt et al. (2002) study was the presentation of the sound. In the current study, both the AC and BC sound was applied bilaterally while only the AC sound was given to both ears in Stenfelt et al. (2002). The use of unilateral stimulation in the Stenfelt et al. (2002) study was motivated by the low transcranial attenuation of BC sound (Stenfelt, 2012). The inter-aural difference would, on average, be close to $0 \mathrm{~dB}$ for frequencies below $1 \mathrm{kHz}$ and be limited by about $10 \mathrm{~dB}$ at higher frequencies (Stenfelt, 2012). However, the inter-subject spread in BC transcranial attenuation data is great and there could be important inter-aural differences for some of the participants in the Stenfelt et al. (2002) study. Such inter-aural differences could affect the perception of loudness due to differences in binaural loudness summation. According to Zwicker et al. (1991), for narrow-band noises similar to that used in the current study and the 
study by Stenfelt et al. (2002), inter-aural differences of 4 dB or less did not produce a significant different binaural loudness summation than equal binaural stimulation. They also reported that for levels of $40 \mathrm{~dB}$ SPL and above, it was only at the highest level (90 dB SPL) that the binaural loudness summation showed any level dependence. Also Scharf (1969) indicated that binaural loudness summation of narrow-band signals was almost constant with level. This is further in line with recent data of Sivonen et al. (2006) who also reported minimal influence from the stimulation level on binaural loudness summation. Consequently, even though the inter-aural level difference with BC stimulation was not controlled in the Stenfelt et al. (2002) study, the influence from binaural loudness summation seem to be negligible and those data can be compared with the current study.

Even so, the finding that $\mathrm{AC}$ and $\mathrm{BC}$ stimulation produced different loudness functions could not be explained easily. For example, such results indicate that the vibration level of the basilar membrane as a function of stimulation level was different for AC than for BC sound. However, the results in the literature indicate that the vibration level of the basilar membrane as a function of stimulation level was similar for AC and BC stimulation. One such example was AC-BC cancellation experiments, where an AC tone perceptually canceled a BC tone. In such experiments, no effect of the stimulus level on the relative amplitude and phase settings at the cancellation point was reported (Khanna et al., 1976; Stenfelt, 2007). It should be mentioned that in the study of Khanna et al. (1976), they reported a remaining $2^{\text {nd }}$ harmonic at cancellation for frequencies below $2 \mathrm{kHz}$; this $2^{\text {nd }}$ harmonic increased with increasing stimulation level. Although this may suggest differences in the vibration pattern of the basilar membrane for AC and BC stimulation, the remaining $2^{\text {nd }}$ harmonic could also be explained by distortion in the BC transducer (Stenfelt, 2007). However, there are other plausible explanations for the results found here and in the Stenfelt et al. (2002) study, for example (1) 
non-linear sound transmission, (2) differences in efferent control for AC and BC sound, and (3) multi-sensory loudness integration.

\subsection{Non-linear sound transmission}

The non-linearity could originate in the stimulation transducer, the transmission pathway, and/or in the cochlea. For AC stimulation, the non-linearity of the stimulation transducer was considered negligible for stimulation levels up to $100 \mathrm{~dB}$ SPL. Also, the sound transmission through the outer and middle ear was expected to be linear (Merchant et al., 1996) with the exception of the acoustic reflex that may have affected the transmission at the higher levels for the low-frequency sound (Neumann et al., 1996; Zhao et al., 2010). It has been shown that the acoustic reflex may be elicited at stimulation levels down to 50 to $60 \mathrm{~dB}$ SPL (Goodman et al., 2006; Zhao et al., 2010). Consequently, the acoustic reflex may influence the sound transmission for the low-frequency sound at levels above $50 \mathrm{~dB}$ SPL. There are only limited data on the influence of the acoustic reflex on BC sound transmission in the human (Stenfelt, 2010) but animal studies have indicated it to be similar to AC stimulation (Irvine, 1976).

The transmission of BC sound in the human skull was reported linear for the levels used in this study (Håkansson et al., 1996; Stenfelt et al., 2005a). The BC transducer used a balanced technology that reduced the non-linear distortion compared with conventional BC transducers such as the Radioear B71. As a consequence, the total harmonic distortion was less than 3\% even at the highest BC stimulation levels, which was in line with the distortion analysis provided in Håkansson (2003). This means that the stimulation one octave above the stimulation frequency (at the $2^{\text {nd }}$ harmonic) was about $30 \mathrm{~dB}$ below the level of the stimulation frequency. But, because the sensitivity to BC sound was approximately $12 \mathrm{~dB}$ better at $1.5 \mathrm{kHz}$ than at $0.75 \mathrm{kHz}$, the effective stimulation due to the distortion in the $\mathrm{BC}$ transducer at the $2^{\text {nd }}$ harmonic was some $15-20 \mathrm{~dB}$ below that of the fundamental stimulation. 
Even if this distortion added to the perceived loudness, according Moore et al. (1997) the effect was less than $1 \mathrm{~dB}$.

Therefore, neither BC skull transmission nor the BC transducer caused a significant distortion component to influence the loudness function. However, at the highest BC levels, for some subjects, the dynamic force exceeded 5 Newtons $(134 \mathrm{~dB}$ re $1 \mu \mathrm{N})$ which was greater than the static force pressing the BC transducer against the mastoid. Such a condition would be associated with significant distortion overtones that could significantly increase the perceived loudness.

The processes in the cochlea are non-linear, including the action of the outer hair cells that influence basilar membrane vibration and the processes within the inner hair cells that transform those vibrations into neural signals. The AC sound transmission is straight-forward involving the outer and middle ear before producing a sound pressure in the scala vestibuli in the cochlea (Aibara et al., 2001). The BC sound transmission is more complex involving several frequency-dependent pathways that integrate to produce a vibration of the basilar membrane (Stenfelt, 2011; Stenfelt et al., 2005b). However, even if the BC sound transmission is more complex than the AC sound transmission, there are no indications that BC sound have a different level growth function of the basilar membrane vibration than AC sound. According to the above, it is not likely that the finding of a general difference in the loudness function between AC and BC sound originate in non-linearities, neither in the transducers, sound transmission pathways, nor the cochlea.

\subsection{Efferent control}

The perception of loudness involves central processes (Neuhoff et al., 1999) and the neural transmission may be affected by efferent control mechanisms (Stenfelt et al., 2009). For example, it has been shown that the auditory evoked amplitude of the neural activity in the 
brainstem is modulated by the cognitive load (Sörqvist et al., 2012). Consequently, if the BC sound influences the efferent control differently than the AC sound, the loudness function difference may be related to changes in the top-down control for either AC or BC sound. However, there are no indications in the literature that such differences between AC and BC stimulation exist. Even so, one such efferent control mechanism is the acoustic reflex mentioned in the previous section. However, the acoustic reflex influences the sound transmission to the cochlea and its effect would change the basilar membrane vibration. Such a change of basilar membrane vibration would influence the amplitude and phase setting in AC-BC cancellation experiments. As described previously, no effect of stimulation level were reported in AC-BC cancellation experiments and the acoustic reflex was not likely the major origin for the different loudness functions found in this study. Furthermore, the acoustic reflex affects the sound transmission at higher stimulus levels, and the differences between the slopes of the loudness functions for AC and BC stimulation were largest with low-level stimuli.

\subsection{Multi-sensory loudness integration}

As noted in the previous section, loudness perception includes central processing. Consequently, other sensory inputs may add to the perception of the auditory stimuli with the integrated perception being greater than auditory alone. One such example is that the combination of auditory and tactile stimulation facilitates detection of a stimulation (Wilson et al., 2010a). Another example is that the combination of auditory and tactile stimulation result in greater perception of loudness than auditory stimulation alone (Wilson et al., 2010b). As a result, the steeper loudness function with BC stimulation compared with AC stimulation may be caused by integration of multi-sensory input present with BC stimulation but absent with AC stimulation. 
A relevant question is then what multi-sensory stimulation is available with BC stimulation but not with AC stimulation? Vibrotactile excitation was present during BC stimulation, especially at lower frequencies and at higher levels (Brinkmann et al., 1983). For example, the approximate vibrotactile threshold level for $750 \mathrm{~Hz}$ shown in Fig $2 \mathrm{~b}$ indicated that it was only at the highest levels for the low-frequency stimulation that the tactile perception could influence the loudness perception with BC stimulation. However, the difference between AC and BC stimulated loudness functions were seen already at low levels. Moreover, the difference between the slopes of the AC and BC stimulated loudness functions were present at the higher frequencies. If the vibrotactile excitation only influenced the loudness perception at levels above the vibrotactile threshold, it was not the sole explanation for the finding of different loudness functions for AC and BC sound.

Another modality that could be excited during BC stimulation was the vestibular system. This system also was reported to be evoked at low frequencies and high levels (Sheykholeslami et al., 2001). Therefore, it did not explain the findings here as statistically significant difference between the loudness functions for $\mathrm{AC}$ and $\mathrm{BC}$ stimulation was found at the low stimulation levels.

\subsection{Individual results}

The analysis has so far focused on the average results from the measurements. In Fig 4, the individual data for the loudness level differences were included. As can be seen, there were individual differences and a few participants even showed steeper loudness functions for AC sound than for BC sound (negative loudness level difference values). In Fig 4a, three participants had an overall steeper loudness function with AC than with BC sound for the low-frequency stimulation, and in Fig 4b, four participants had an overall steeper loudness function with $\mathrm{AC}$ than with $\mathrm{BC}$ sound for the high-frequency stimulation. Consequently, the 
majority (17 of 20 for low-frequency sound and 16 of 20 for high-frequency sound) showed a steeper loudness function for stimulation with BC than with AC sound.

The analysis of the individual results were further done to investigate if the same individuals who had the greatest loudness level differences for AC and BC low-frequency stimulation (upper part of Fig 4a) also had the greatest loudness level differences for the high-frequency stimulation (upper part of Fig 4b). This was done by ranking the participants according to their overall AC-BC loudness level differences for the low-frequency and high-frequency stimulation. According to this ranking, the three participants showing the greatest overall ACBC loudness level difference for low-frequency stimulation were the same three participants showing the greatest overall AC-BC loudness level difference for high-frequency stimulation. Moreover, when a median split was made for the ranking of the low-frequency and highfrequency AC-BC loudness level difference, eight of the ten participants showing the greatest differences for the low-frequency stimulation were among the ten participants showing the greatest differences for the high-frequency stimulation. Consequently, there were consistencies between the participants' loudness functions at low and high-frequency stimulation.

There were only limited parameters that could be further analyzed looking for origins for the participants variations of the AC-BC loudness level differences. All participants had normal hearing and no analysis was done regarding hearing thresholds. The age range in the study was limited to 18 to 42 years, and it was difficult to draw firm conclusion relating the influence of age on the results. However, the average age of the ten participants with the greatest differences according to Fig 4a was 30.7 years while the average age of the other ten participants was 30.5 years. Consequently, for the limited age range in this study, there was no indication that age influenced the results. 
The participants consisted of 12 males and 8 females. Due to the limited numbers, it is not possible to make a thorough analysis of the impact of gender on the results. Even so, if the low-level slope of the loudness function $\left(k_{l o}\right)$ for the low-frequency sound was compared between stimulation modalities, the $k_{l o \_A C} / k_{l o \_B C}=0.79$ for the females while the same ratio was 0.92 for the males. Moreover, six of the eight participating females were among the ten highest ranked for the difference between AC and BC loudness levels, while eight of twelve males were among the ten lowest ranked. Consequently, the results indicate that females have greater differences between $\mathrm{AC}$ and $\mathrm{BC}$ loudness functions than males. However, due to the limited number of participants and non-matched groups, this indication needs further research before any conclusions can be drawn.

\subsection{Clinical applications}

Even if the current study could not explain the origin of the difference between the loudness functions for AC and BC stimulation, the results in this and previous studies have clinical relevance for the use of BC hearing aids, for example the BAHA (Snik et al., 2005). Although statistically significant, the ratio of the slopes for the AC and BC stimulated loudness functions was about 0.9. Consequently, the total effect was limited. Even so, one aim of prescriptions algorithms for hearing aids is to restore the perception of loudness. In the light of the current result, the compression function of the gain for BC hearing aids should be different than for AC hearing aids. It should be noted that this may not be the same as restoring audibility, and the current result may suggest that the maximum output of BC hearing aids should be limited rather than reducing the gain at lower levels. However, such a limited maximum output level of BC hearing aids is already accomplished by the limited maximum output level of BC hearing aids in general. 


\section{Conclusions}

The loudness function for low-frequency BC noise bursts was statistically significantly steeper than the loudness function for low-frequency AC noise bursts. The ratio of the slopes of the loudness functions for the two stimulation modalities was 0.88 . When the stimulation was a high-frequency noise burst, the ratio of the AC and the BC loudness function slopes was 0.92 , but was only statistically significant at the low stimulation levels. These results were similar to the differences between the loudness functions for $\mathrm{AC}$ and $\mathrm{BC}$ stimulation reported by Stenfelt et al. (2002). Different loudness estimation procedures were used in the current study (adaptive categorical loudness scaling) and in Stenfelt et al. (2002) (loudness balancing). No conclusive evidence for the origin of the difference was found. It was speculated that the difference partly could originate in multi-sensory stimulation with BC sound that was not present with AC stimulation. It was also plausible that the effect was a summation of several smaller effects as loudness integration of multi-sensory stimulation, spectral loudness summation due to distortion of the BC transducer, and binaural loudness summation due to unequal inter-aural BC stimulation. 


\section{Acknowledgements}

This study was supported by Stingerfonden. 


\section{Captions}

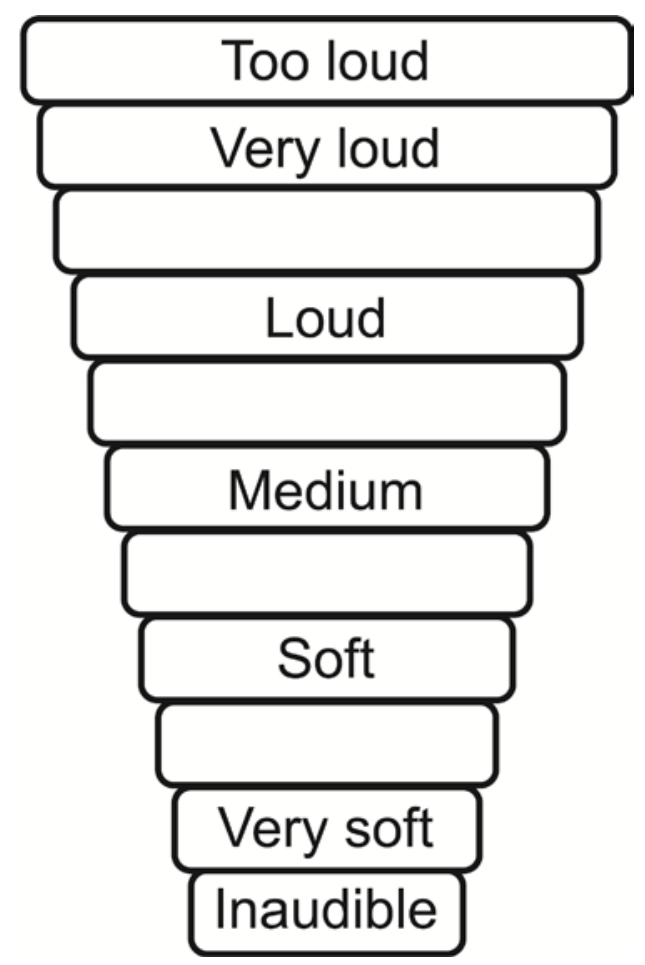

Fig 1: The 11-item scale used for the loudness estimation. The participant clicked on one of the items subsequent to each sound stimulation to rate the loudness. 


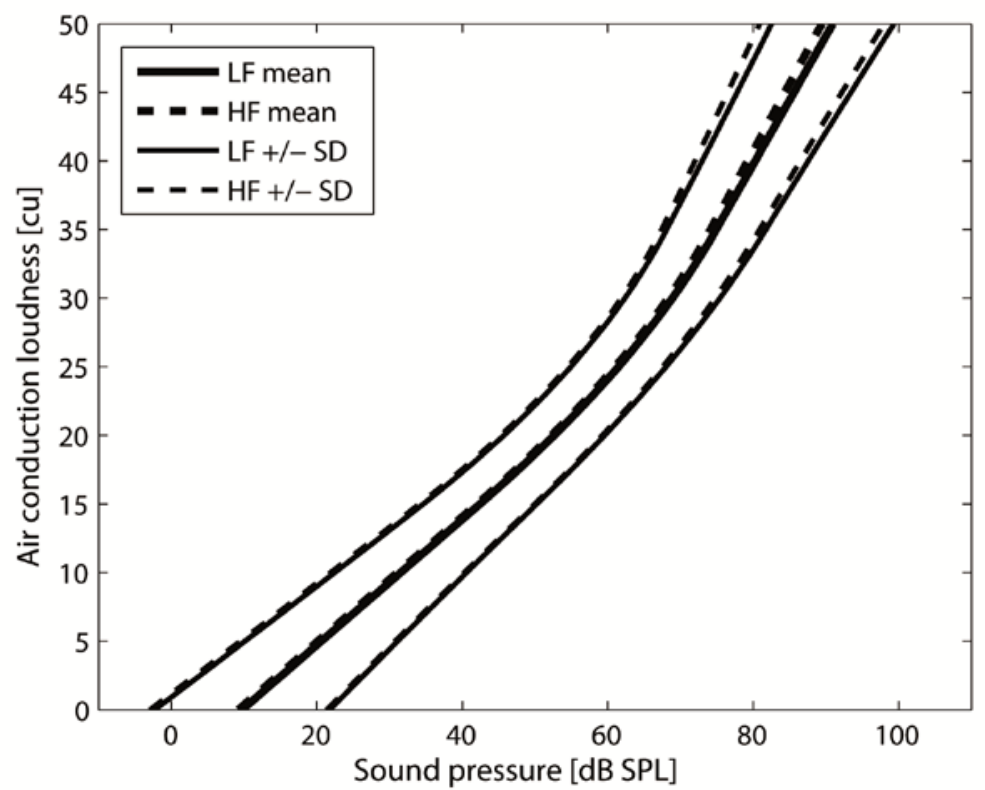

b)

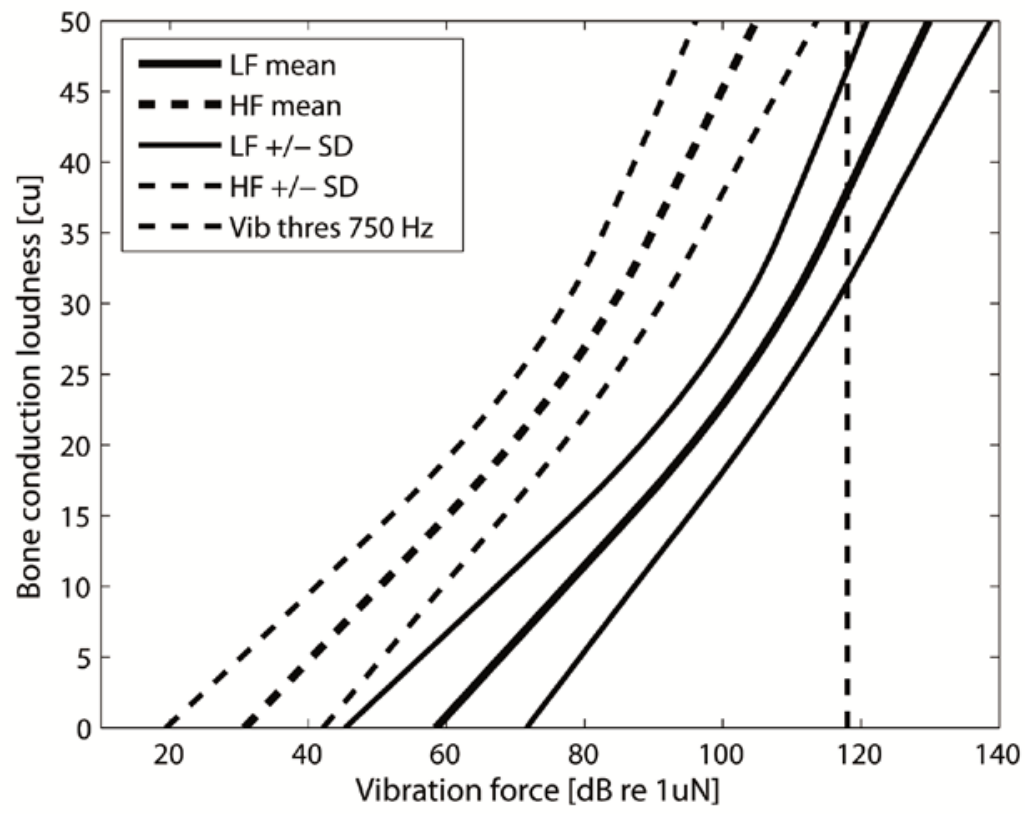

Fig 2: Loudness functions from the categorical loudness scaling procedure for (a) AC stimulation in sound pressure level, and (b) BC stimulation in force level. The loudness functions are based on the averaged parameters $k_{l o}, k_{h i}$, and $L_{c u t}$, and the average loudness functions for the low-frequency stimulation are shown as solid thick 
lines and for the high-frequency stimulation as dashed thick lines. The averages plus/minus one SD according to the parameter estimates are shown as thin lines using the same line properties as the averaged data. The vibrotactile threshold for a $750 \mathrm{~Hz}$ BC tone is indicated in (b) by a vertical dashed line. 


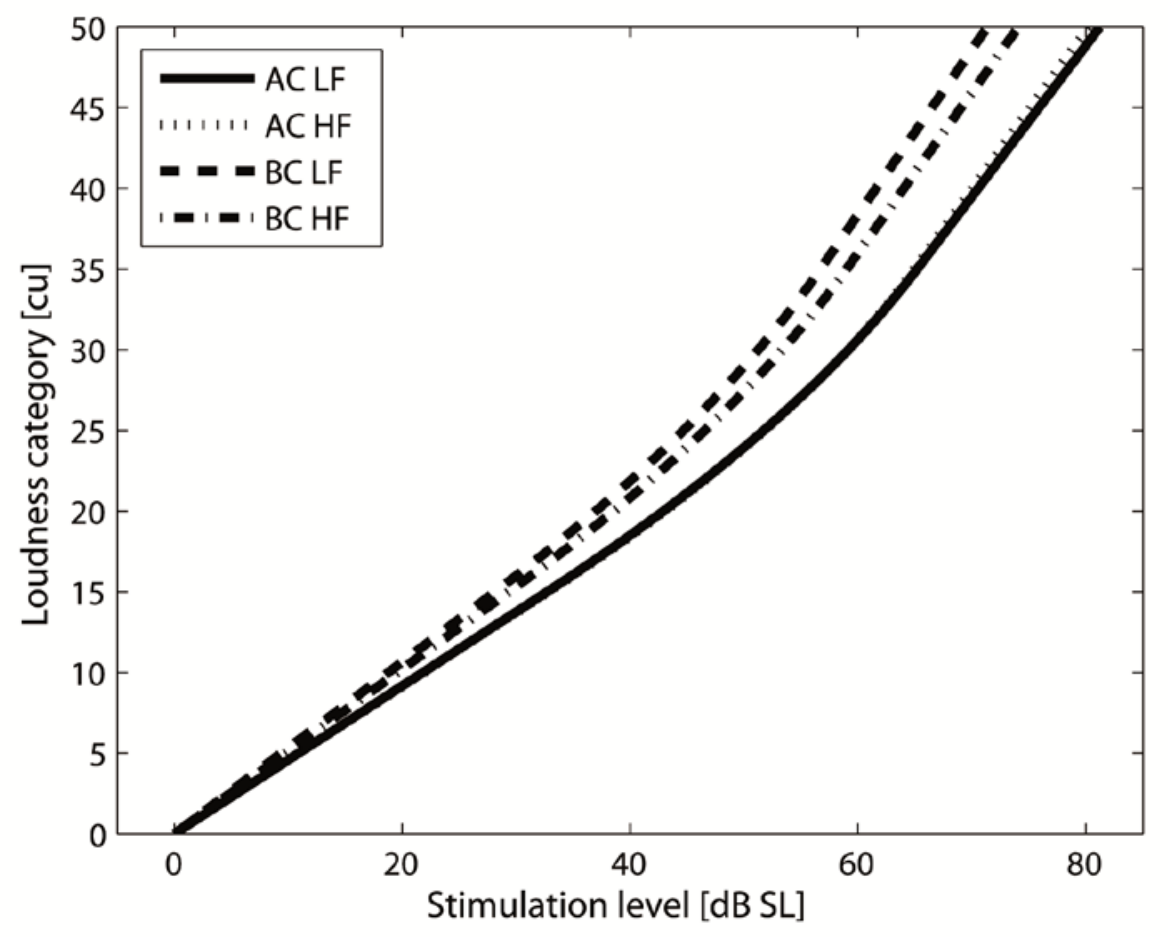

Fig 3: The loudness functions based on the averaged parameters $k_{l o}, k_{h i}$, and $L_{c u t}$, according to Equation 1 in relation to the stimulation level in sensation level (dB SL) for the four stimulations. The result with the AC low-frequency stimulation is shown as a solid line, the result with the AC high-frequency stimulation as a dotted line, the result with the BC low-frequency stimulation as a dashed line, and the result with the BC high-frequency stimulation as a dash-dotted line. 
a)

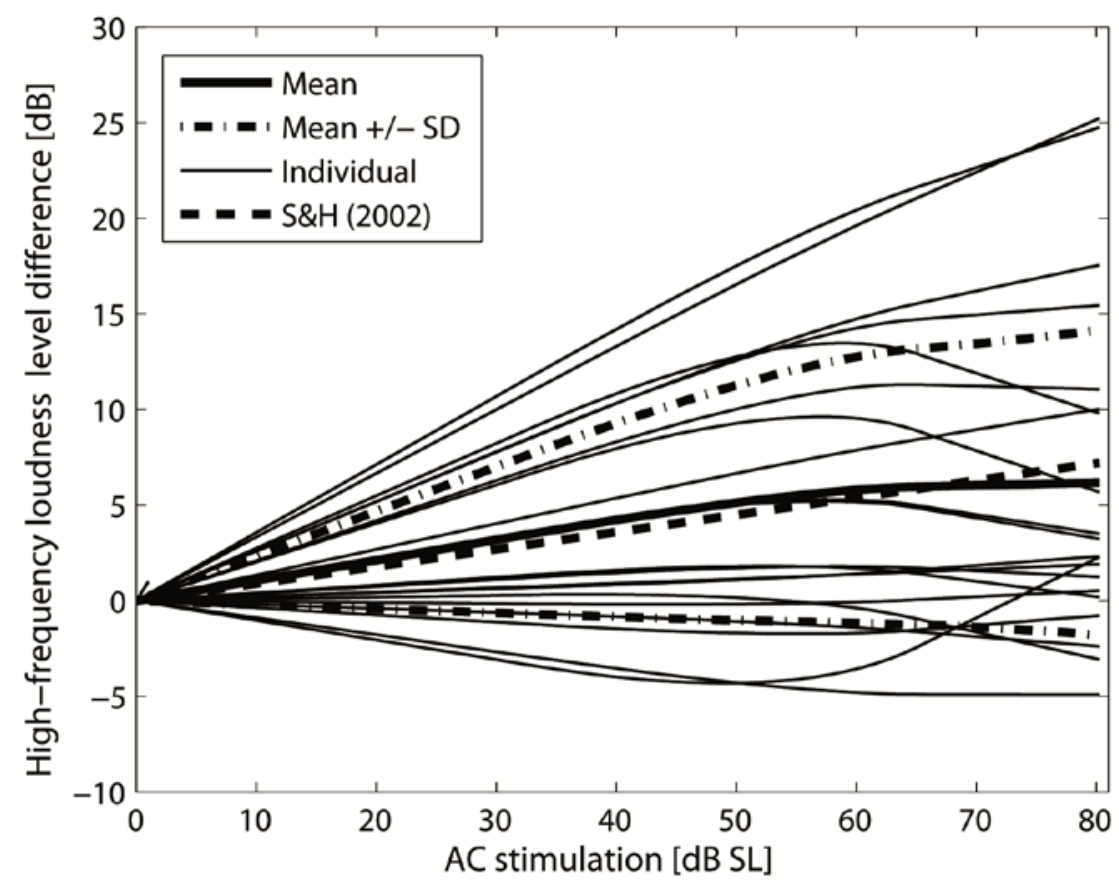

b)

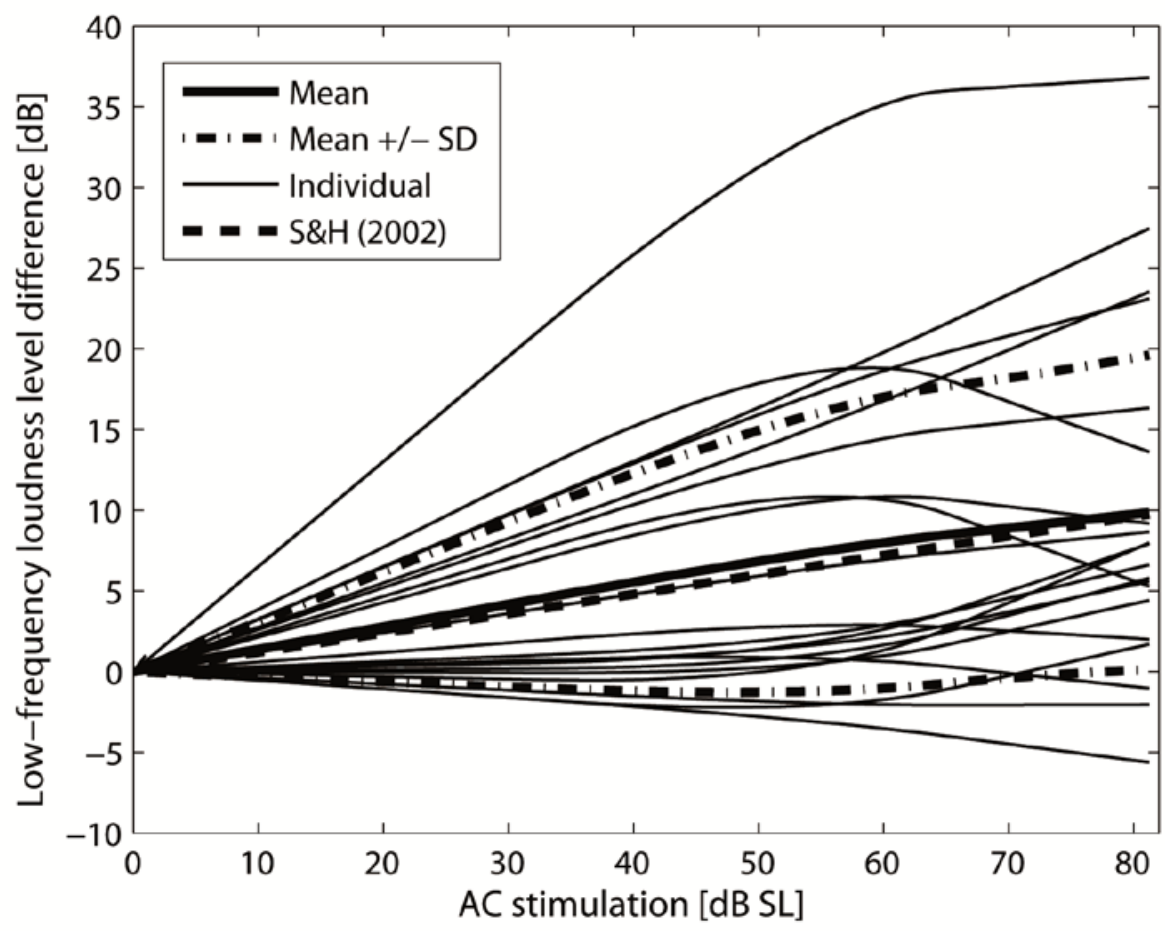

Fig 4: The loudness level differences between the AC and BC stimulation are shown for the low-frequency stimulation in (a) and for the high-frequency stimulation in (b). The loudness level differences are related to the stimulation level of the AC sound. The 
average loudness level differences are shown with a thick solid line, the averages plus/minus one standard deviation are shown with dash-dotted lines, and the individual loudness level differences are shown with thin solid lines. Also included in the figures are the average loudness level differences between $\mathrm{AC}$ and $\mathrm{BC}$ stimulation for $750 \mathrm{~Hz}$ (a) and $4 \mathrm{kHz}$ (b) from Stenfelt et al. (2002). 


\section{References}

Aibara, R., Welsh, J., Puria, S., Goode, R. 2001. Human middle ear sound transfer function and cochlear input impedance. Hearing Research 152, 100-109.

Beattie, R. 1998. Normative wave V latency-intensity functions using the EARTONE 3A insert earphone and the Radioear B-71 bone vibrator. Scand. Audiol. 27, 120-126.

Brand, T., Hohmann, V. 2002. An adaptive procedure for categorical loudness scaling. J Acoust Soc Am 112, 1597-604.

Brinkmann, K., Richter, U. 1983. Determination of the normal threshold of hearing by bone conduction using different types of bone vibrators. Part I. Audiol Akust (Germany) 22, 62-85.

Goodman, S., Keefe, D. 2006. Simultaneous measurement of noise-activated middle-ear muscle reflex and stimulus frequency otoacoustic emissions. Journal of the Association for Research in Otolaryngology 7, 125-139.

Haeff, A., Knox, C. 1963. Perception of ultrasound. Science 139, 590-592.

Hosoi, H., Imaizumi, S., Sakaguchi, T., Tonoike, M., Murata, K. 1998. Activation of the auditory cortex by ultrasound. The Lancet 351, 496-7.

Håkansson, B. 2003. The balanced electromagnetic separationtransducer: Anew bone conduction transducer. J. Acoust. Soc. Am. 113, 818-825.

Håkansson, B., Carlsson, P., Brandt, A., Stenfelt, S. 1996. Linearity of sound transmission through the human skull in vivo. J. Acoust. Soc. Am. 99, 2239-2243.

IEC:60318-1. 2009. International Electrotechnical Commission, Simulators of human head and ear.

Part 1: Ear simulator for the measurement of supra-aural and circumaural earphones, Geneva.

Irvine, D. 1976. Effects of reflex middle-ear muscle contractions on cochlear responses to boneconducted sound. Audiology 15, 433-444. 
ISO:389-1. 1998. International Organization for Standardization, Reference zero for the calibration of audiometric equipment. Part 1: Reference equivalent threshold sound pressure levels for pure tones and supra-aural earphones, Geneva.

ISO:389-3. 1994. International Organization for Standardization Reference zero for the calibration of audiometric equipment. Part 3: Reference equivalent threshold force levels for pure tones and bone vibrators, Geneva.

ISO:389-8. 2004. International Organization for Standardization, Reference zero for the calibration of audiometric equipment. Part 8: Reference equivalent threshold sound pressure levels for pure tones and circumaural earphones, Geneva.

ISO:16832. 2006. International Organization for Standardization Loudness scaling by means of categories, Geneva.

Khanna, S.M., Tonndorf, J., Queller, J. 1976. Mechanical parameters of hearing by bone conduction. J. Acoust. Soc. Am. 60, 139-154.

Kim, N., Homma, K., Puria, S. 2011. Inertial bone conduction: Symmetric and anti-symmetric components. Journal of the Association for Research in Otolaryngology 12, 261-279.

Merchant, S., Ravicz, M., Rosowski, J. 1996. Acoustic input impedance of the stapes and cochlea in human temporal bones. Hearing Research 97.

Moore, B., Glasberg, B., Baer, T. 1997. A model for the prediction of thresholds, loudness, and partial loudness. Journal of the Audio Engineering Society 45, 224-240.

Neuhoff, J., McBeath, M., Wanzie, W. 1999. Dynamic frequency change influences loudness perception: a central, analytic process. Journal of Experimental Psychology. Human Perception and Performance 25, 1050-1059.

Neumann, J., Uppenkamp, S., Kollmeier, B. 1996. Detection of the acoustic reflex below $80 \mathrm{~dB}$ HL. Audiology and Neuro-Otology 1, 359-369.

Nishimura, T., Okayasu, T., Uratani, Y., Fukuda, F., Saito, O., Hosoi, H. 2011. Peripheral perception mechanism of ultrasonic hearing. Hearing Research 277, 176-183. 
Scharf, B. 1969. Dichotic Summation of Loudness. J. Acoust. Soc. Am. 45, 1193-1205.

Sheykholeslami, K., Kermany, M., Kaga, K. 2001. Frequency sensitivity range of the saccule to boneconducted stimuli measured by vestibular evoked myogenic potentials. Hearing Research 160.

Sivonen, V., Ellermeier, W. 2006. Directional loudness in an anechoic sound field, head-related transfer functions, and binaural summation. J. Acoust. Soc. Am. 119, 2965-2980.

Snik, A.F., Mylanus, E.A.M., Proops, D.W., Wolfaardt, J.F., Hodgetts, W.E., Somers, T., Niparko, J.K., Wazen, J.J., Sterkers, O., Cremers, C.W.R.J., Tjellström, A. 2005. Consensus statements on the BAHA system: Where do we stand at present? Annals of Otology, Rhinology and Laryngology 114, Supplementum 195:1-12.

Stenfelt, S. 2007. Simultaneous cancellation of air and bone conduction tones at two frequencies: extension of the famous experiment by von Békésy. . Hearing Research 225, 105-116.

Stenfelt, S. 2011. Acoustic and physiologic aspects of bone conduction hearing. Advances in OtoRhino-Laryngology 71, 10-21.

Stenfelt, S. 2012. Transcranial attenuation of bone conducted sound when stimulation is at the mastoid and at the bone conduction hearing aid position. Otology and Neurotology 33, 105114.

Stenfelt, S. 2010. Effect of the stapedius reflex on air conduction and bone conduction transmission in the human. Hearing Research 263, 243.

Stenfelt, S., Håkansson, B. 2002. Air versus bone conduction: An equal loudness investigation. Hearing Research 167, 1-12.

Stenfelt, S., Goode, R.L. 2005a. Transmission properties of bone conducted sound: Measurements in cadaver heads. J. Acoust. Soc. Am. 118, 2373-2391.

Stenfelt, S., Goode, R. 2005b. Bone conducted sound: Physiological and clinical aspects. Otology and Neurotology 26, 1245-1261. 
Stenfelt, S., Reinfeldt, S. 2007. A model of the occlusion effect with bone-conducted stimulation. International Journal of Audiology 46, 595-608.

Stenfelt, S., Rönnberg, J. 2009. The Signal-Cognition interface: Interactions between degraded auditory signals and cognitive processes. Scandinavian Journal of Psychology 50, 385-393.

Stenfelt, S., Puria, S., Hato, N., Goode, R.L. 2003. Basilar membrane and osseous spiral lamina motion in human cadavers with air and bone conduction stimuli. Hearing Research 181, 131-143.

Sörqvist, P., Stenfelt, S., Rönnberg, J. 2012. Working memory capacity and visual-verbal cognitive load modulate auditory-sensory gating in the brainstem: Towards a unified view of attention. Journal of Cognitive Neuroscience 24, 2147-2154.

Watanabe, T., Bertoli, S., Probst, R. 2008. Transmission pathways of vibratory stimulation as measured by subjective thresholds and distortion-product otoacoustic emissions. Ear and Hearing 29, 667-673.

Wilson, E., Reed, C., Braida, L. 2010a. Integration of auditory and vibrotactile stimuli: Effect of frequency. J. Acoust. Soc. Am. 127, 3044-3059.

Wilson, E., Braida, L., Reed, C. 2010b. Perceptual interactions in the loudness of combined auditory and vibrotactile stimuli. J. Acoust. Soc. Am. 127, 3038-3043.

von Békésy, G. 1932. Zur Theorie des Hörens bei der Schallaufnahme durch Knochenleitung. Ann. Physik 13, 111-136.

Zhao, W., Dhar, S. 2010. The effect of contralateral acoustic stimulation on spontaneous otoacoustic emissions. Journal of the Association for Research in Otolaryngology 11, 53-67.

Zwicker, E., Zwicker, U. 1991. Dependence of binaural loudness summation on interaural level differences, spectral distribution, and temporal distribution. J. Acoust. Soc. Am. 89, 756-764. 